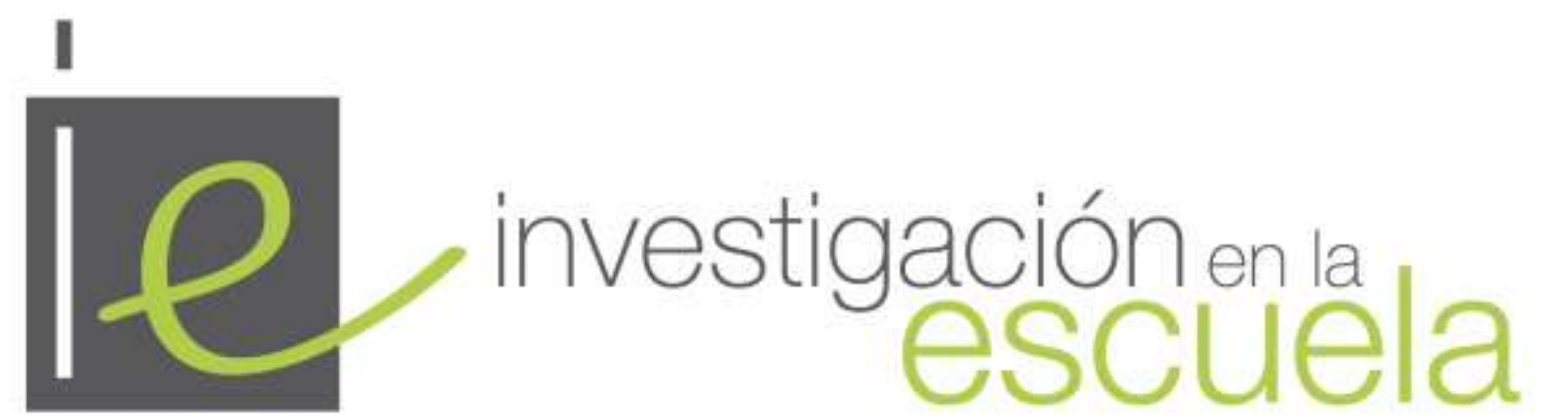

Revista internacional de investigación e innovación educativa

Número 95

15 de octubre de 2018

ISSN 2443-9991

\title{
La innovación en la enseñanza de las ciencias también es una cuestión política e ideológica. I: La génesis y fundamentos del Proyecto IRES
}

Gabriela Cattani Delord', Rafael Porlán y Gleny Duro Guiarães ${ }^{2}$

Universidad de Sevilla ${ }^{1}$, Pontificia Universidad Católica de Río Grande del Sur ${ }^{2}$

España, Brasil

Citación: Delord, G. C, Porlán, R. y Duro Guiarães, G. (2018). La innovación en la enseñanza de las ciencias también es una cuestión política e ideológica. I: la génesis y fundamentos del Proyecto IRES. Investigación en la Escuela, 95, 1-14. Recuperado de: http://www.investigacionenlaescuela.es/articulos/R95/R95-1

Resumen: Este artículo, el primero de una serie de dos, se basa en la idea de que todo discurso innovador en la enseñanza de las ciencias (y por extensión en la escuela) tiene un trasfondo político e ideológico con consecuencias sociales. Teniendo esto en cuenta, se analiza el discurso del Proyecto IRES (Investigación y Renovación Escolar) a través de las entrevistas a sus cuatro fundadores y del análisis de tres artículos publicados por ellos. Se utilizó el Análisis de Discurso (AD) como método analítico y los resultados ponen de manifiesto que el proyecto tiene una ideología alternativa generada en el contexto antifranquista y que su origen parte del Principio de Investigación (como cualidad común al conjunto de los seres humanos) enriquecido por las influencias teóricas del constructivismo, la complejidad y la teoría crítica.

Palabras clave: "Innovación"; "análisis del discurso"; "enseñanza de las ciencias: proyecto IRES"; "investigación en la escuela".

Innovation in the science education is also a political and ideological issue. I: The genesis and foundations of the IRES Project 
Abstract: This article, the first of a series of two, is based on the idea that all innovative discourse in the science education (and by extension in the school) has a political and ideological background with social consequences. Taking this into account, the discourse of the IRES Project (Research and Renewal of the School) is analyzed through interviews with its four founders and the analysis of three articles published by them. Speech Analysis (AD) was used as an analytical method and the results show that the project has an alternative ideology generated in the anti-Franco context and that its origin is based on the Research Principle (as a quality common to all human beings) enriched by the theoretical influences of constructivism, complexity and critical theory.

Key words: "Innovation"; "discourse analysis"; "science education"; "IRES project"; "research in the school".

\section{L'innovation dans l'enseignement des sciences est aussi une question politique et idéologique. I : La genèse et les fondements du projet IRES}

Resumè: Cet article, le premier d'une série de deux, est basé sur l'idée que tout discours novateur dans l'enseignement des sciences (et par extension dans l'école) a un fond politique et idéologique avec des conséquences sociales. En prenant cela en compte, le discours du projet IRES (Recherche et Renouvellement de l'Ecole) est analysé à travers des entretiens avec ses quatre fondateurs et l'analyse de trois articles publiés par eux. L'analyse de la parole (AD) a été utilisée comme méthode analytique et les résultats montrent que le projet a une idéologie alternative générée dans le contexte anti-franquiste et que son origine est basée sur le principe de recherche (qualité commune à tous les êtres humains) enrichi par les influences théoriques du constructivisme, de la complexité et de la théorie critique.

Mots-clés: "Innovation"; "analyse du discours"; "éducation scientifique"; "projet IRES"; "recherche à l'école".

\section{Introducción}

En la década de los ochenta el cambio político en España influyó en cierto redescubrimiento de la renovación pedagógica (Cuesta y Fernández Cuadrado, 2000). Según Duarte (2015), en la universidad, la presencia del autoritarismo se diluía y los estudiantes, junto con los profesores más activos, caminaban de la mano. En aquel momento, se fue extendiendo un discurso de cambio escolar frente al discurso franquista. Al mismo tiempo, se produce un boom de publicaciones y proyectos curriculares innovadores, entre ellos el Proyecto IRES - en adelante P. IRES-, del que trata este estudio. Para Varela (2007), la sociedad española del momento estaba inclinada al cambio y a la construcción de un nuevo sistema educativo surgido de la necesidad política del contexto histórico. Para Stenhouse (1987), refiriéndose en este caso a Reino Unido, la mayoría de los proyectos de aquel momento se centraban más en la calidad del proceso que en sus resultados cuantitativos.

Actualmente, también se habla de innovación y de cambio pedagógico. Sin embargo, una parte del discurso innovador actual se centra más en una cuestión práctica de técnicas y recursos, que en la cuestión política de transformar el sistema educativo y la sociedad. Continuamente aparecen nuevas propuestas (no tan nuevas) en la prensa, muchas de ellas provenientes del ámbito anglosajón, cuando hasta hace poco el cambio de la escuela no tenía interés periodístico. Las hay que proponen hacer una innovación más tecnológica (Cabello, 2006) o que proponen la clase invertida (Strayer, 2012; Toppo, 2011), el aprendizaje basado en problemas (Duch, Gron y Allen, 2001), el aprendizaje a través de retos (Jou, Hung, Lai, 2010) o el aprendizaje por proyectos (Railback, 2002), entre otras. Todas ellas autoconsideradas nuevas e innovadoras, aunque muchas tienen ya viejos antecedentes en nuestro país. 
Según Hannan y Silver (1999), el concepto de innovación cambia según la institución y la posición del sujeto que lo defiende (profesor, gestor, político...). La innovación es, por tanto, un concepto polisémico y ambiguo (García, 2010) que puede ser entendido y aplicado de distintas formas, pudiendo perseguir, en cada caso, objetivos diferentes. No hay un consenso sobre lo que es innovador. Los autores mencionados afirman que, si las instituciones económicas y financieras están involucradas en la búsqueda de mejoras y de nuevos caminos educativos, entonces analizar las innovaciones debe significar tratar de comprender las ideologías relacionadas. Por lo tanto, la innovación debe ser abordada desde un punto de vista menos ingenuo, pues puede responder a objetivos con fuerte intencionalidad política y económica, ya que no todo lo que se dice innovador es realmente transformador.

Para identificar estas cuestiones nos hemos apoyado en la teoría del AD de M. Pêcheux (1995), desde la que hemos analizado un proyecto de larga trayectoria e influencia como es el P. IRES. Este artículo, el primero de dos, analiza la génesis y los fundamentos del proyecto (Grupo Investigación en la Escuela, 1991) y el siguiente se centrará en su evolución hasta nuestros días. Ambos son parte de un estudio más complejo del que presentaremos aquí solo una parte. Otros análisis ya han sido publicados (Delord, Porlán y Harres, 2017) o están en proceso de revisión.

\section{Marco teórico}

Cuando hablamos de discurso también hablamos de ideología. Según M. Pêcheux (1995) el discurso y la ideología son inseparables, y toda ideología posee efectos concretos en la sociedad. Por tanto, lo innovador es ideológico y tiene fines y consecuencias.

Existen distintas corrientes teóricas sobre el discurso (Delord, 2015). En nuestro caso nos hemos apoyado en las investigaciones sociales de los años ochenta sobre temas emergentes como la sexualidad, el género, la etnicidad y el ateísmo, relacionadas con lo que Fraser (2003) llama las luchas a favor del reconocimiento de la diferencia. Estos investigadores buscaban explicaciones haciendo preguntas del tipo: ¿Por qué no se cambia?, ¿por qué se siguen reproduciendo las pautas del discurso tradicional o convencional? En general, los analistas entienden que el discurso es una dimensión crucial en el establecimiento de los vínculos y las relaciones y, por tanto, en el mantenimiento o evolución de las dinámicas sociales (Santander, 2011).

En nuestro caso, situamos el discurso innovador de la enseñanza de las ciencias en el centro del análisis. Nuestra intención ha sido identificar cuál es el trasfondo político/ideológico del discurso del P. IRES, qué influencias ha recibido y cómo ha sido condicionado por el contexto histórico y social. Para realizar este análisis desde la perspectiva del AD hemos tenido en cuenta las siguientes dimensiones: La Formación Discursiva (FD) (lo que se dice), la Formación Ideológica (FI) (la ideología que subyace) y la Posición de los Sujetos (PS) (los pronunciamientos concretos). Para ello proponemos una tabla de componentes o categorías que caracterizan lo que vamos a llamar Formación Innovadora/Alternativa y Formación Innovadora/Neoliberal (Tabla 1).

Tabla 1

Componentes de la Formación Innovadora/Alternativa y de la Formación Innovador/Neoliberal

FD Innovadora y FI Alternativa

Fines vinculados al desarrollo personal y a un modelo socio-ambiental justo.

- Contenidos conceptuales globalizados vinculados a problemas sociales y
FD Innovadora y FI Neoliberal

Fines vinculados a la autorrealización individual a través del emprendimiento y la competencia.

- Contenidos asociados a competencias 
ambientales relevantes. Importancia de los procedimientos y valores alternativos a los socialmente dominantes.

- Metodología crítica e investigativa. Se enseña con, desde y para hacer evolucionar las posiciones de los que aprenden.

- Evaluación no sancionadora y formativa. Se cuestiona el papel de la calificación como instrumento de poder.

- La innovación afecta a todas las dimensiones mencionadas y a su coherencia. medibles.

- Metodologías activas. Uso de nuevas tecnologías.

- Evaluación basada en la medición de competencias, sancionadoras y selectiva. No se cuestiona la calificación.

- La innovación se centra en el cambio metodológico y en nuevos recursos técnicos.

Cómo podemos ver en la tabla 1, el componente metodológico puede parecer próximo en los dos enfoques de la innovación, sin embargo, los fines, los contenidos y la evaluación/calificación muestran profundas diferencias.

\section{Metodología}

El AD está situado en el paradigma cualitativo interpretativo (Valles, 2000). En la primera etapa del análisis se deben elegir los textos y documentos adecuados para seleccionar trozos literales que nos informen sobre la problemática que se pretende investigar. Es decir, unidades de información en las que el sujeto, a través de su discurso, se posiciona dentro de un contexto. Después de tener el corpus discursivo, el analista establece las dimensiones básicas mencionadas: La FD explícita, en nuestro caso el P. IRES se autodefine como innovador y alternativo, la Posición de los Sujetos analizados y la FI que finamente se infiere.

Como en toda investigación, la metodología debe ser coherente con los problemas de investigación. Si éstos no se plantean en términos de inferir el trasfondo ideológico (social, político, económico...), el AD no es pertinente y habría que buscar apoyo en otros análisis (Santander, 2011). Es decir, el AD no es aplicable en todos los casos. centrales:

Pues bien, nuestro problema de investigación se concreta en las siguientes cuestiones

¿Cuál es la ideología que está detrás del discurso innovador en el ámbito de la enseñanza de las ciencias del Proyecto IRES?

¿En qué contexto histórico se formuló?

¿Qué otros discursos influyeron?

¿Cuáles son sus fines, fundamentos y propuestas?

Las dos fuentes de información elegidas han sido la entrevista semiestructurada y en profundidad (Gil, 2007), realizada a cada uno de los fundadores, y tres artículos publicados por ellos en los que explican las características del proyecto (García y Porlán, 2000; García Díaz, 2005; Merchán y García, 1994). Estos materiales han sido objeto de recortes discursivos (unidades de información) que han permitido identificar las dimensiones del AD.

Según Santander (2011), para guiar el proceso de análisis, la pregunta orientadora ha de ser siempre: ¿Qué busco en el texto? El AD no pretende cuantificar la información sino entender el problema y buscar los significados que informan sobre él. En el corpus del texto podemos encontrar mucha información, sin embargo, para no perdernos, para discriminar entre aquello que interesa y aquello que no, siempre es aconsejable preguntarse una y otra vez: ¿Qué estoy buscando en el texto? 
Como estrategia elegimos el estudio de caso único, que implica un proceso de indagación para comprender en profundidad un fenómeno particular (una clase, un centro, un proyecto innovador...). Para Stake (1998), lo relevante de este tipo de estudios es lo específico y complejo de un caso singular.

En relación con las entrevistas, y en base a las cuestiones centrales ya mencionadas, las preguntas orientadoras que han guiado la sesión con cada fundador han sido:

a) ¿Cuáles fueron los intereses que llevaron a diseñar el Modelo de Investigación en la Escuela (MIE) y el Proyecto IRES?

b) ¿En qué contexto educativo, social y politico fue diseñado?

c) ¿Cuáles son los fines del MIE para la enseñanza de las ciencias?

d) ¿Por qué este modelo didáctico y no otro?

e) ¿Cómo analiza y valora la enseñanza de las ciencias mayoritaria?

f) ¿Cómo desde el Proyecto IRES se ha intentado favorecer e impulsar el cambio en la enseñanza de las ciencias $y$ en la escuela?

g) ¿Cuáles han sido los obstáculos que ha tenido el proyecto para extenderse y generalizarse?

b) ¿Cuáles han sido las estrategias utilizadas para sortear dichos obstáculos y hacer avanzar el Proyecto IRES a lo largo de los años?

En este artículo analizamos las preguntas a, b, c, d y e. Las restantes se analizarán en el artículo siguiente.

Los sujetos desarrollaron, de forma natural, su punto de vista sobre las preguntas, con las mínimas intervenciones del entrevistador y en un entorno agradable y relajado (Delord, 2017). Posteriormente las entrevistas se transcribieron literalmente. Tanto las transcripciones como los artículos fueron interrogados a partir de las cuestiones centrales mencionadas, seleccionando los recortes discursivos relevantes e identificando las diferentes Posiciones Sujetos que a continuación se presentan. Dichos recortes fueron codificados con la letra A cuando se obtuvieron de las transcripciones de las entrevistas, seguida del número correspondiente a cada autor $(1,2,3$ y 4$)$, o con las letras Art cuando se obtuvieron de los artículos, seguidas también del número de orden de cada uno (1, 2 y 3 ). Por razones de espacio, en cada PS se muestra solo una parte representativa de las unidades de información identificadas.

\section{Análisis discursivo}

\section{Posición de los Sujetos I: Antifranquismo, cambio social y cambio pedagógico}

Como ya hemos dicho, el AD integra el discurso en su contexto histórico, pues el discurso es el efecto de la relación del sujeto con la lengua y con la historia (Orlandi, 2012, p. 48). El análisis de las entrevistas y los artículos nos llevaron a estudiar el período de la dictadura de Franco en España, para comprender algunas de sus afirmaciones.

De cara a posibles lectores de ámbitos diferentes al español, resumimos brevemente dicho contexto. España vivió una guerra civil entre 1936-1939 que dejó más de 500.000 víctimas y abrió el camino para el franquismo totalitario y la extrema derecha. En febrero de 1936, fue elegido democráticamente el Frente Popular, coalición formada por partidos socialistas, comunistas y republicanos (Buades, 2006), que prometía una política de regeneración política y de inclusión social con medidas consideradas radicales por la derecha: la reforma agraria, el estado laico, la amnistía a los presos políticos, la alfabetización de los campesinos, la educación pública y la emancipación de las mujeres. El profundo desacuerdo de la derecha con este programa provocó un golpe de estado liderado por Franco (1892-1975). Con el golpe, el país pasó a vivir bajo un discurso ultraconservador, nacionalista y totalitario. 
En un estado de esta naturaleza, los autores del P. IRES vivieron su escolarización y su carrera universitaria. Estaba suprimida la libertad de expresión, de reunión y de asociación, y el no cumplimiento de estos preceptos acarreaba tortura, prisión e incluso asesinato.

Después de la muerte del dictador (1975), Juan Carlos I asumió el reinado de España y el país inició un proceso de democratización, amnistiando a los presos políticos e iniciando la transición hacia un sistema de monarquía parlamentaria. Durante este período, el denominado Movimiento de Renovación Pedagógica gana fuerza en el ámbito educativo. Entre los colectivos docentes que pertenecían a él destaca, en el ámbito de la ciudad de Sevilla (ciudad donde se origina el proyecto), el Colectivo Andaluz de Pedagogía Popular (CAPP), del cual formaban parte los fundadores del P. IRES a través del Seminario de Didáctica de las Ciencias Naturales. Por lo tanto, antes de diseñar el IRES, los fundadores ya actuaban pedagógicamente contra el gobierno dominante de la época.

él.

Visto este contexto general, analizaremos ahora las Posiciones Sujetos de los autores frente a

Los fundadores resaltan la influencia de la transición democrática y de la victoria electoral del Partido Socialista en la emergencia de un ambiente social de ilusión y de cambio:

...Para entender el P. IRES hay que tener en cuenta el contexto que habia en España en los años 80, en el que estaba madurando un proceso de transición democrática (...). En 1982, es la primera vez que se da un cambio de verdad, cuando gana el partido socialista después de más de 40 años de dictadura. Hubo una explosión en todos los sentidos, una explosión cultural tremenda (...) y apareció en el mundo de la educación una gran cantidad de innovaciones. Estábamos muy ilusionados. Estas innovaciones parecian nuevas, pero bien analizadas, muchas tenian sus raices en la riquisima tradición de los años 30, que fue interrumpida por la dictadura (A.1, PS1).

También señalan cómo sus experiencias están marcadas por la represión y por su posición política antifranquista.

Trabajé en la universidad desde el año 74 hasta el 76, estaba haciendo mi tesis de doctorado. Nosotros éramos, de forma muy clara, antifranquistas. En el 76 llegó a la universidad un catedrático muy fascista y me puso en la calle (A.3, PS1).

Al principio, ya de profesores, tuvimos muchos problemas para trabajar en la universidad de forma innovadora. La Dirección de la Escuela de Magisterio se esforzaba, por ejemplo, en poner mesas y sillas fijadas al suelo, dificultando el trabajo en grupo. Estábamos en minoría y teniamos que luchar para hacer los cambios. Estuvimos muy controlados, pero teníamos muchos alumnos a nuestro favor (A.2, PS1).

\section{Posición de los Sujetos 2: Apertura a influencias vinculadas con el Principio de Investigación}

En esta segunda PS hemos identificado que el Principio de Investigación no fue elaborado de manera aislada por los fundadores. Otros investigadores ya venían trabajando en la misma idea. Por tanto, sus concepciones recibieron importantes influencias que las confirmaron y desarrollaron.

Por una parte, la formación biológica de tres de ellos les llevó a reflexionar sobre la potencialidad didáctica de la investigación científica y sobre las cualidades de nuestra especie para explorar el entorno. Por otro lado, su pertenencia al Movimiento de Renovación Pedagógica les aportó influencias pedagógicas, especialmente en relación con la idea del aprendizaje por investigación:

Cuando empezamos (...) nuestra reflexión era: si los científicos aprenden investigando, ¿será que todas las personas pueden aprender así? O sea, llevar el método científico a la escuela, claro que no con las mismas exigencias, pero utilizando el mismo proceso básico, pues creíamos que era una característica común a todas las personas (A.4, PS2). 
El ser humano se adapta bien a las situaciones de incertidumbre, exploración y buisqueda de soluciones, aprendiendo de la novedad y presentando una tendencia a buscarla. Al conjunto de estos procesos podemos llamarle también investigación. Así, la investigación podría ser considerada como una estrategia básica de conocimiento y actuación en la realidad, propia de nuestra especie, común al conjunto de la población bumana y con un claro valor adaptativo (Art.2, PS2).

Entonces comenzamos a reflexionar, por un lado, sobre cómo se forma el conocimiento científico y, por otro, sobre lo que se sabia de la Didáctica de las Ciencias, consultando a autores que ya investigaban el aprendizaje por investigación. Con todo esto fuimos dando forma a la idea de Investigación en la Escuela (A.4, PS2).

Junto con (autores) fundamos el primer Seminario de Didáctica de las Ciencias en Andalucía, (...) éramos profesores que voluntariamente queríamos trabajar en la Didáctica de las Ciencias. Eso era muy importante porque en el 82 se publicó un libro en español que cambió nuestras vidas, un libro de André Giordan, y empezamos a compartir la didáctica francesa, que pasó a ser nuestra línea de referencia, piagetiana y cognitiva (A.3, PS2).

...en los años 80 estábamos en una explosión del Movimiento de Renovación Pedagógica de Freinet, Freire, un poco más adelante Tonucci. En especial Freinet con la Educación Popular (A.1, PS2).

Fueron dos patas, en una Giordan y en la otra Tonucci. En el momento en que comenzamos a leer los primeros libros de ellos fue un impacto, descubrimos cosas que no sabíamos y confirmamos otras que intuíamos, a partir de ahi empezamos a investigar todo ese campo del constructivismo y la teoría crítica que están descritos en el P. IRES. Giordan hablaba en su libro de investigación en clases de ciencias de secundaria y Tonucci de infantil (...), y asi fuimos construyendo nuestras referencias (A.3, PS2).

Un día nos encontramos con el libro de Tonucci "A los 3 años se investiga". Recuerdo que fue en casa de (autor), quien dijo: mira el libro que he encontrado. Nos quedamos impresionados porque decía lo que estábamos sospechando. Fue como si descubriéramos que no estábamos solos, ese libro nos impactó mucho. Otro fue el de Giordan ("La Enseñanza de las Ciencias"), que describia la investigación en la Didáctica de las Ciencias, que también nos impresionó, porque eran personas importantes en el área escribiendo lo que ya estábamos pensando. Entonces dijimos: es que no estamos tan locos (A.4, PS2).

Estas influencias sirvieron para superar dos extremos sobre la idea de investigación: el descubrimiento espontáneo y la investigación dirigida única y cerrada; y empezamos a considerar las ideas de los estudiantes como la base de un enfoque constructivista del aprendizaje por investigación.

Era necesario buscar fundamentos de cómo se aprende. Eso no surge solo de nuestra reflexión, sino también de las influencias que nos estaban Ilegando, especialmente desde Francia que ya estaban trabajando con las representaciones de los alumnos, y de Inglaterra, sobre el 83, que estaban llegando escritos sobre las concepciones y sobre cómo se aprende. Esto llega antes al campo de la enseñanza de las ciencias que a otros (A.1, PS2).

Entonces nos fuimos distanciando de esos 2 polos: la investigación espontaneísta y la dirigista. Tenemos que reconocer que también habia otros autores con ese pensamiento (A.1, PS2).

Todo esto llevó a los fundadores a replantearse la idea del papel del docente. El principio de investigación ya no afectaba solo al aprendizaje de los estudiantes, sino también, y por coherencia, al aprendizaje docente. Los modelos de investigación-acción y la metáfora del docente como investigador confirmaron posteriormente esta convicción.

En España éramos los únicos que escribiamos sobre esto. Entonces concluimos que, si el alumno aprende por investigación, el profesor también debe aprender así. Entonces deberíamos hacer formación docente que incluyera la idea de investigación. Pasamos de la idea del niño como investigador a la del profesor como investigador y a la de una escuela investigadora (A.4, PS2).

...empezamos a alimentarnos de la investigación-acción, a través de Elliot y Stenhouse, y todo eso colaboró con las ideas teóricas del IRES, o sea, no fue sólo nuestra convicción (A.1, PS2). 
El P. IRES pretende fortalecer una visión compleja del cambio educativo, articulando la investigación de equipos de docentes críticos para generar y consolidar una cultura profesional alternativa (...) favoreciendo una práctica profesional que sigue el modelo de profesor-investigador (Art.2, PS2).

Finalmente, esto permitió que los fundadores del proyecto, a través de las Jornadas de Investigación en la Escuela -que empezaron a organizar desde 1983-, a las que fueron invitados los autores mencionados, pasaran a formar parte de una comunidad investigadora internacional que trabajaba con principios parecidos.

En esta perspectiva, estábamos desarrollando cada año las Jornadas de Investigación en la Escuela aqui en Sevilla, que reunian a todas las personas del área y traíamos invitados a nuestros autores de referencia. Entonces conviviamos con ellos y fuimos creando una especie de comunidad internacional que compartíamos este modelo (A.4, PS2).

En resumen, los autores fueron influenciados por las lecturas de Giordan (1982), Tonucci (1977) y Elliot (1990), entre otros, lo que significó que el Principio de Investigación fue el resultado de la interacción entre sus concepciones y las lecturas de estos textos que las confirmaron y las hicieron respetables, al provenir de autores de prestigio ("no estamos tan locos"). No sabemos cuál hubiera sido el discurso si las interacciones hubieran sido con otros autores o sobre otras variables del proceso de enseñanza-aprendizaje.

\section{Posición de los Sujetos 3: Elaboración de un Modelo Didáctico y Curricular innovador, alternativo y fundamentado}

El MIE tiene desde el principio un trasfondo ideológico para los autores, pues pretende proporcionar el espíritu crítico y los valores necesarios para cuestionar las ideas dominantes de la enseñanza tradicional y su carácter hegemónico, así como para desarrollar una cultura escolar alternativa.

...es un modelo alternativo pues persigue valores educativos (autonomia, respeto a la diversidad, igualdad, solidaridad, cooperación) (...) que consideramos alternativos a los socialmente dominantes (Art.2, PS3).

Los supuestos que defendemos rompen con todo eso, rompen con la estructura tradicional, que es como una bola absolutamente coherente que va en una dirección muy fuerte y hegemónica (...). La mayoría de la población piensa que la enseñanza tradicional no es una opción, sino que es la enseñanza que tiene que ser y no hay cuestionamientos sobre eso (...) la escuela tradicional es una hegemonía porque siempre fue asi y nadie se cuestiona que puedan existir otras maneras de enseñar (A.4, PS3).

Pretendemos introducir en el sistema escolar mayores niveles de diversidad, libertad y autonomía que favoreqcan la construcción de una cultura escolar alternativa a la tradicional (Art.2, PS3).

Por otro lado, como hemos visto en la PS anterior, las innumerables influencias recibidas provocaron una mayor profundización teórica. Así el constructivismo, la complejidad (Morin, 1977) y la teoría crítica comienzan a formar parte del bagaje conceptual de los fundadores, pero sin perder la capacidad de formular principios prácticos para la acción.

Un proyecto de estas características busca su fundamentación en aportaciones científicas, ideológicas y políticas, sin trasladar de forma mecánica dichas aportaciones como recetas para la enseñanza; antes bien se trata de integrar esa diversidad de aportaciones y referentes, reelaborándolas en un nuevo marco, de carácter específicamente didáctico (Art.2, PS3).

Entonces con el tiempo identificamos que la idea de investigación tenía que estar más fundamentada y así fuimos incorporando el constructivismo, la teoría de sistemas y la teoría crítica, o sea otras teorias que la completaban y le daban... (A.4, PS3).

Pero el IRES tiene tres grandes fundamentos teóricos, uno es el constructivismo, porque ayuda a entender cómo se aprende, otra es la complejidad, pues los procesos de enseñanza y aprendizaje son complejos, diversos y 
sistémicos y no deben ser simplificados. En especial nos influyeron las lecturas de Morin. Por último, la teoría crítica. Son los tres ríos que alimentan el IRES (A.1, PS3).

El camino de construcción del IRES, su potencialidad, fue hacer una tentativa de convertir las perspectivas constructivista, compleja y crítica en teorías operativas, en principios prácticos que el profesor pudiese tener como referentes prácticos, es lo que llamamos Principios Didácticos, sobre todo el de Investigación (....). Yo creo que esa es la clave de que el IRES fuese tan potente y transformador, pues todo el bagaje teórico fue convertido en principios orientadores muy próximos a la práctica (A.1, PS3).

En definitiva, el P. IRES propone un modelo didáctico alternativo y fundamentado: el Modelo de Investigación en la Escuela (MIE). Este modelo ha sido desarrollado tanto para el aprendizaje del alumnado como para la formación del profesorado. Según los documentos originales del P. IRES, este modelo debe favorecer el cambio de la enseñanza de las ciencias y de la escuela tradicional, que tiende a uniformizar a los estudiantes en un único modelo de pensamiento y conducta (aprendizaje de la sumisión):

Este modelo es una propuesta para intentar desarrollar en las escuelas concepciones y prácticas distintas a la cultura escolar conocida, cultura que perdura y se reproduce en sus objetivos básicos a pesar de las reformas de las leyes. Un único modelo de enseñanza para un único modelo ideológico, no cumpliendo un papel democrático" (Grupo Investigación en la Escuela, 1991, p.19). Más detalles del MIE pueden consultarse en Grupo Investigación en la Escuela (1991), Delord, Porlán y Harres (2017) y Delord (2017).

Pero el MIE, en base a todo lo anterior, presenta también una propuesta curricular alternativa, fundamentalmente desarrollada en el ámbito de la enseñanza de las ciencias. Los contenidos escolares (denominados conocimiento escolar) son entendidos desde una perspectiva transformadora y crítica distinta a los contenidos del currículum tradicional, seleccionando asuntos de interés para los alumnos y problemas socioambientales relevantes en el plano local y general. El cómo es entendido como un proceso constructivo e investigativo tanto de carácter individual como grupal.

Para la enseñanza de las ciencias, nuestra postura supone entender los contenidos como un instrumento de desarrollo personal y de cambio social. (...). Proponemos que el conocimiento escolar tenga sentido, sea significativo y funcional para la persona que aprende (Art.1, PS3).

... no se trata de alcanzar una respuesta ya dada, de descubrir una verdad preexistente, sino de crear nuestras propias respuestas (Art.1, PS3).

El eje que articula el qué y el cómo son los problemas de investigación, se trata de problemas abiertos y complejos, socioambientales, que deben conectar con sus intereses y sus preocupaciones y deben permitir también movilizar contenidos culturales significativos y socialmente relevantes (Art.1, PS3).

La construcción del conocimiento es, a su vez, un proceso individual y social, que se produce simultáneamente en ambos planos. De hecho, se propone la necesidad de superar el dualismo individuo-cultura proponiendo una visión más integradora, en la que las personas y el contexto, las creencias personales y las sociales coevolucionen (Art.1, PS3).

En resumen, en el análisis realizado de las entrevistas y los artículos hemos caracterizado tres PS de los fundadores del P. IRES: a) Una clara posición política de origen antifranquista y a favor de un profundo cambio social y pedagógico; b) Una posición pedagógica inicial basada en el Principio de Investigación (como transposición de la investigación científica) y abierta a innumerables influencias externas; y c) Una posición pedagógica y curricular elaborada, fundamentada y práctica que pretende ser una alternativa general al modelo de enseñanza tradicional.

Los autores, a través de estas posiciones, están insertos en una Formación Ideológica Alternativa, pues se posicionan de forma contraria a la ideología dominante, marcada en el momento inicial por el franquismo. Desde esta posición desarrollan, a través del MIE, una Formación Discursiva Innovadora que fomenta fines favorables al desarrollo de ciudadanos y ciudadanas para el 
cambio social, promoviendo a través de la investigación de los problemas relevantes de la sociedad, una nueva visión de los elementos curriculares.

\section{Conclusiones}

Reiteramos que todo discurso pedagógico tiene un trasfondo ideológico ya que discurso e ideología son interdependientes. Además, siguiendo la teoría de M. Pêcheux (1995), algunos discursos llamados innovadores pueden presentar Posiciones Sujetos que siguen fomentando la ideología conservadora/neoliberal dominante.

Teniendo esto en cuenta hemos analizado el P. IRES y nos hemos formulado las siguientes preguntas de investigación: ¿Cuál es la ideología que está detrás del discurso innovador del P. IRES?, ¿en qué contexto histórico se formuló?, ¿qué otros discursos influyeron? y ¿̇cuáles son sus fines, fundamentos y propuestas? A través de las entrevistas a los fundadores y de 3 artículos seleccionados podemos responder que el $\mathrm{P}$. IRES tiene una ideología de fondo desarrollada desde el antifranquismo y de carácter antiautoritario y liberador. Que el proyecto y el MIE han sido construidos a partir del Principio de Investigación enriquecido por innumerables aportaciones y que se sintetizan en tres teorías organizadoras: el constructivismo como forma de explicar la génesis individual, social e histórica del conocimiento; la complejidad como una visión del mundo superadora de reduccionismos y simplificaciones; y la teoría crítica como forma de entender las relaciones de clase entre conocimiento, poder y conducta. Todo ello trasladado al plano curricular, formulando una propuesta de conocimiento escolar y profesional con identidad propia, que parte de las concepciones y experiencias de los propios sujetos y que basa su construcción en la investigación de problemas socio-ambientales (en el caso de los estudiantes) y profesionales (en el caso de los docentes).

Por último, y volviendo al principio, el análisis de las propuestas innovadoras nos debe llevar a saber si contribuyen a la ideología dominante o a una ideología alternativa de cambio social. Estas son cuestiones aún poco investigadas en el ámbito de la enseñanza de las ciencias. Para nosotros, sin embargo, son cuestiones importantes que deben ser consideradas, pues, si queremos una sociedad más justa e igualitaria, las prácticas llamadas innovadoras no deben contribuir a mantener la ideología dominante.

La auténtica innovación solo será posible, desde nuestro punto de vista, cuando la sociedad, los alumnos, los docentes y los investigadores perciban que la cultura escolar tradicional, más allá de los fines declarados, atiende a los objetivos de los gobiernos y de las clases a las que representan (Althusser, 1980). Así, los sectores dominantes quieren garantizar a través de la escuela, las convenciones sociales, políticas, económicas y culturales que atienden a sus intereses: disciplina, orden, jerarquía, mérito, competencia y reproducción de los ideales conservadores o/y neoliberales (Orlandi, 1987). Por lo tanto, este "currículo oculto" (Torres, 1991), que casi nadie ve, es uno de los pilares que sostiene las crisis económicas, la desigualdad social y la crisis energética, entre otros problemas relevantes actuales.

Según Cuesta (1998), la población sólo va a presionar hacia un cambio educativo de fondo cuando ocurra una crisis social, como ocurrió en la década de los ochenta, cuando el P. IRES, así como otros muchos, fue puesto en marcha. Sin embargo, creemos que también, como nos enseña Paulo Freire (1991), los oprimidos sólo dejan de ser oprimidos el día en que se dan cuenta (toman conciencia) de que son oprimidos. Por tanto, crisis estructural y liberación personal son dos partes imprescindibles de un mismo proceso de transformación social y escolar.

\section{Referencias}


Althusser. L. (1989). A transformação da filosofia seguido de Marx e Lênin perante Hegel. São Paulo: Ediciones Mandacaru.

Buades, E. (2006). Revolución en España. Madrid: Contraimagen.

Cabello, R. (2006). "Yo con la computadora no tengo nada que ver". Un estudio de las relaciones entre los maestros y las tecnologías informáticas en la enseñanza. Buenos Aires: Prometeo Libros.

Cuesta, R. (1998). Clio en las aulas. La enseñanza de la Historia en España entre reformas, ilusiones y rutinas. Madrid: Akal.

Cuesta, F. y Fernández Cuadrado, M. (2000). El proyecto Cronos para la enseñanza de las Ciencias Sociales: Balance y perspectivas de un itinerario pedagógico. En VV.AA. Aspectos didácticos de las Ciencias Sociales, 14 (pp.99-135). Instituto de Ciencias de la Educación de la Universidad de Zaragoza.

Delord, G.C. (2015). Análise do Discurso com M. Pêcheux. En G. Gleny (Coord.). Re-significando os labirintos da pesquisa qualitativa, (pp. 95-110). Porto Alegre: EDIPUCRS,

Delord, G.C. (2017). La innovación en la enseñanza de las ciencias. La identificación de obstáculos ideológicos: estudio de caso del Proyecto IRES (Tesis doctoral). Universidad de Sevilla.

Delord, G.C., Porlán, R. y Harres, J.B. (2017). La importancia de los proyectos y redes innovadoras para el avance de la enseñanza de las ciencias: el caso de un profesor de la Red IRES. Revista Eureka sobre Enseñanza y Divulgación de las Ciencias, 14(3), 653-665. DOI:10498/19514.

Duarte, O. (2015). La enseñanza de la historia en la educación secundaria: innovación, cambio y continuidad (Tesis doctoral). Universidad de Sevilla.

Duch, B., Gron, S. y Allen, D. (2001). The power of problem-based learning. A practical "bow to" for teaching undergraduate courses en any discipline. Delawere: University of Delaware.

Elliot, J. (1990). Implications of classroom research for professional development. En E. Hoyle y J. Megarry (ed.). Professional development of teachers, (pp. 308-324). London: Kogan Page

Fraser, N. (2003). Nuevas reflexiones sobre el reconocimiento. New Left Review, 4, 107-120.

Freire, P. (1991). Pedagogia do oprimido. Rio de Janeiro: Paz e Terra.

García, F.F. y Porlán, R. (2000). El proyecto IRES. Biblio 3W. Revista Bibliográfica de Geografía y Ciencias Sociales, 5 (205). Disponible en http://www.ub.edu/geocrit/b3w-205.htm.

García, P.S. (2010). Inovações, mudanças: Por que elas não acontecem na escola? São Paulo: LCT Editora.

García Díaz, J. E. (2005). Complejidad y construcción del conocimiento. Actas del VII Congreso Internacional sobre Investigación en la Didáctica de las Ciencias. Número Extra. Enseñanza de las Ciencias.

Barcelona.

Gil, A.C. (2007). Como elaborar projetos e pesquisa. Sao Paulo: Atlas.

Giordan, A. (1982). La Enseñanza de las Ciencias. Madrid: Siglo XXI.

Grupo Investigación en la Escuela (1991). Proyecto curricular IRES. Doc. I, II, III, IV. Sevilla: Díada.

Hannan, A. y Silver, H. (1999). La innovación en la Enseñanza Superior: enseñanza, aprendizaje y culturas institucionales. Madrid: Narcea.

Jou, M., Hung, C.K. y Lai, S.H. (2010). Application of Challenge Based Learning Approaches in Robotics Education. International Journal of Technology and Engineering Education, 7(2), 1- 42.

Disponible en: http://ijtee.org/ijtee/system/db/pdf/72.pdf.

Merchán, F.J. y García F.F. (1994). El proyecto IRES: una alternativa para la transformación escolar. Signos. Teoría y práctica de la educación, 13(5) 58-69.

Morin, E. (1977). La Méthode I. La nature de la Nature. París: Editions du Seuil.

Orlandi, E.P. (1987). A linguagem e seu funcionamento: as formas de discurso. Campinas: Pontes.

Orlandi, E.P. (2012). Discurso em análise: sujeito, ideologia e sentido. São Paulo: Pontes Editora.

Pêcheux, M. (1995). Semântica e discurso: uma crítica à afirmação do óbvio. Campinas: Unicamp.

Railsback, J. (2002). Project-based instruction: Creating excitement for learning. Portland, Oregon: 
Northwest Regional Educational Laboratory.

Santander, P. (2011). ¿Por qué y cómo hacer Análisis de Discurso?. Cinta de Moebio. Revista de Epistemología de Ciencias Sociales, 41(1), 207-224. Disponible en www.moebio.uchile.cl/41/santander.html

Stenhouse, L. (1987). Investigación y desarrollo del Currículum. Madrid: Morata.

Stake, R. (1998). Investigación con estudio de caso. Madrid: Morata.

Tonucci, F. (1977). A los tres años se investiga. Barcelona: Avance.

Torres, J. (1991). El curriculum oculto. Madrid: Morata.

Valles, M. (2000). Técnicas cualitativas de investigación social. Madrid: Síntesis.

Varela, J. (2007). Las reformas educativas a debate (1982-2006). Madrid: Morata.

Strayer, J.F. (2012). How learning in an inverted classroom influences cooperation, innovation and task orientation. Learning Environments, 15(2), 171-193.

Toppo, G. (2012). "Flipped" classrooms take advantage of technology. USA Today. Disponible en http://usatoday30.usatoday.com/news/education/story/20111006/flippedclassroomsvirtualtea ching/50681482/1/

\section{Información sobre los autores}

Autor: Gabriela Cattani Delord

Institución: Universidad de Sevilla

Email: gabiccd@hotmail.com

Autor: Rafael Porlán

Institución: Universidad de Sevilla

Email: rporlan@us.es

Autor: Gleny Duro Guiarães

Institución: Pontificia Universidad Católica de Río Grande del Sur (Brasil)

Email: gleny@pucrs.br 


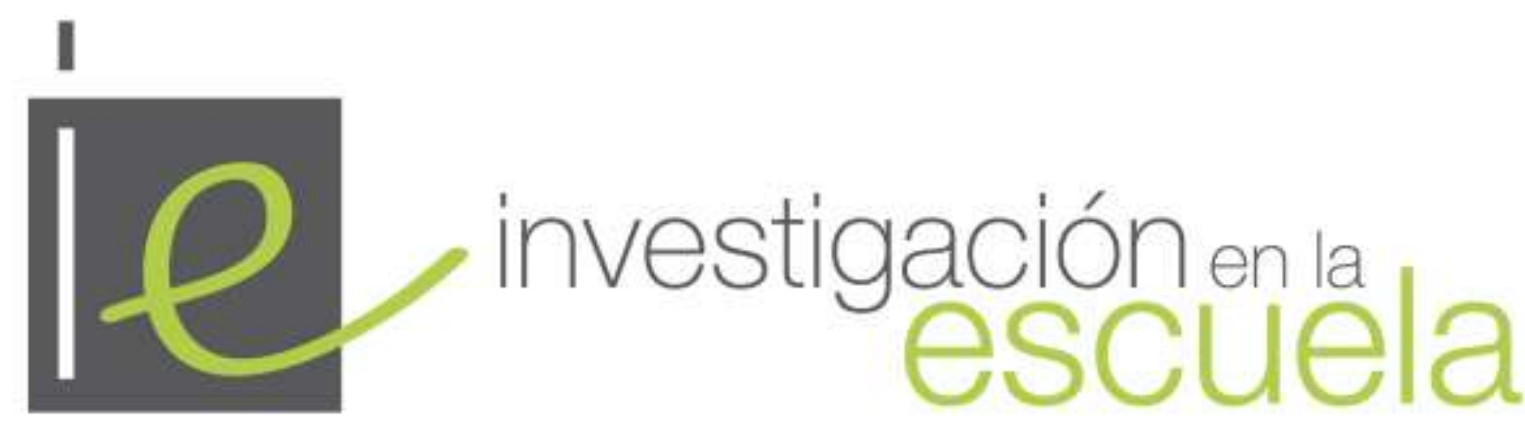

Revista académica evaluada por pares y de acceso abierto

Número 95

15 de octubre de 2018

ISSN 2443-9991

(a)

SOMIERIGHISRESERVEDLOS/as lectores/as pueden copiar, mostrar, y distribuir este artículo, siempre y cuando se de crédito y atribución al autor/es y a Investigación en la Escuela, se distribuya con propósitos no-comerciales, no se altere o transforme el trabajo original. Más detalles de la licencia de CreativeCommons se encuentran en http://creativecommons.org/licenses/by-nc-sa/3.0 Cualquier otro uso debe ser aprobado en conjunto por el autor/es, o Investigación en la Escuela.

ư investigacion-en-la-escuela

Contribuya con comentarios y sugerencias en la web de la revista. Por errores y sugerencias contacte a secretaria@investigacionenlaescuela.es 


\section{Investigación en la escuela}

Consejo de dirección: Ana Rivero García (Universidad de Sevilla), Nicolás de Alba Fernández (Universidad de Sevilla), Pedro Cañal de León (Universidad de Sevilla), Francisco F. García Pérez (Universidad de Sevilla), Gabriel Travé González (Universidad de Huelva), Francisco F. Pozuelos Estrada (Universidad de Huelva)

Dirección: Ana Rivero García y Nicolás de Alba Fernández

Secretaría de edición: Elisa Navarro Medina

\section{Consejo editorial}

José Félix Angulo Rasco. Universidad de Cádiz Rosa Ma Ávila Ruiz. Universidad de Sevilla Pilar AzcárateGoded. Universidad de Cádiz Juan Bautista Martínez Rodríguez. Universidad de Granada

Nieves Blanco García. Universidad de Málaga Fernando Barragán Medero. Universidad de La Laguna José Carrillo Yáñez. Universidad de Huelva José Contreras Domingo. Universidad de Barcelona. Luis C. Contreras González. Universidad de Huelva Ana $\mathbf{M}^{\mathbf{a}}$ Criado García-Legaz. Universidad de Sevilla Rosario Cubero Pérez. Universidad de Sevilla José Ma Cuenca López. Universidad de Huelva Jesús Estepa Giménez. Universidad de Huelva Rafael Feito Alonso. Universidad Complutense (Madrid)

Francisco José García Gallardo. Universidad de Huelva

Soledad García Gómez. Universidad de Sevilla J. Eduardo García Díaz. Universidad de Sevilla
Fernando Hernández Hernández. Universidad de Barcelona

Salvador Llinares Ciscar. Universidad de Alicante Alfonso Luque Lozano. Universidad de Sevilla Rosa Martín del Pozo. Universidad Complutense (Madrid)

José Martín Toscano. IES Fernando Herrera (Sevilla) Jaume Martínez Bonafé. Universidad de Valencia F. Javier Merchán Iglesias. Universidad de Sevilla Emilia Moreno Sánchez. Universidad de Huelva. Rosario Ortega Ruiz. Universidad de Córdoba Antonio de Pro Bueno. Universidad de Murcia Fco. de Paula Rodríguez Miranda. Universidad de Huelva

Pedro Sáenz-López Buñuel. Universidad de Huelva Antoni Santisteban Fernández. Universidad Autónoma (Barcelona)

Emilio Solís Ramírez. Catedrático de IES. $\mathbf{M}^{\mathbf{a}}$ Victoria Sánchez García. Universidad de Sevilla. Magdalena Suárez Ortega. Universidad de Sevilla

\section{Consejo asesor}

Manuel Área Moreira. Universidad de La Laguna Jaume Carbonell. Director Cuadernos de Pedagogía. Barcelona César Coll. Universidad de Barcelona

Christopher Day. Universidad de Nothingham. U.K.

Juan Delval. Universidad Nacional de Educación a Distancia

John Elliott. Universidad de East Anglia. Norwich. U.K.

José Gimeno Sacristán. Universidad de Valencia

André Giordan. Universidad de Paris VII y Ginebra

Francisco Imbernón. Universidad de Barcelona Ángel Pérez Gómez. Universidad de Málaga

Rafael Porlán Ariza. Universidad de Sevilla

Francesco Tonucci. Instituto de Pedagogía del C.N.R. Roma

Jurjo Torres Santomé. Universidad de A Coruña 
